\title{
(Ent-)Kulturalisierungen und (Ent-)Soziologisierungen: Das Soziale, das Kulturelle und die Macht
}

\author{
Ein Kommentar zu Carola Lentz: „Der Kampf um die Kultur: Zur Ent- und \\ Re-Soziologisierung eines ethnologischen Konzepts“"
}

\section{Von Andreas Reckwitz}

Die Sozial- und Geisteswissenschaften sind keine unbeobachteten Beobachter. Die Begriffe, mit denen sie ihre Gegenstände analysieren, bleiben nicht separiert von jenen Semantiken, auf deren Grundlage sich diese Gegenstände jeweils selber beschreiben und damit immer wieder neu verfertigen. Das wissenschaftlich-intellektuelle Feld bildet vielmehr nur einen Knotenpunkt innerhalb der gesellschaftlichen Diskurse und Repräsentationssysteme, zwischen denen keine starren Grenzen existieren. Dass theoretische Begriffe damit - mit Mieke Bal (2002) gesprochen - ,traveling concepts' sind, lässt sich aktuell gut anhand des Konzepts der Kultur studieren. Carola Lentz weist in ihrem Artikel zu Recht auf diese Konstellation hin: Es wäre naiv zu meinen, ,Kultur' - und ihre analytisch engeren und präziseren Korrelate wie Diskurse, Praktiken, Repräsentationssysteme, Semantiken, Deutungsmuster etc. - liefere ausschließlich einen innertheoretischen Impuls zu einer spezifischen Kulturanalyse in den Sozial- und Geisteswissenschaften. Seit den 1990er Jahren gut dokumentierbar, beeinflussen Kategorien der Kultur vielmehr in nicht geringem Maße die Selbstbeschreibung sozialer Gruppen und Institutionen wie auch deren massenmediale Neubeschreibung auf globaler Ebene.

Die Selbstidentifikation von sozialen Gruppen als ,kulturell' findet sich im Falle von manchen sog. ethnischen und religiösen, auch nationalen Kollektiven ebenso wie in der Markierung von kulturellen Differenzen zwischen ganzen Weltreligionen oder Kulturkreisen, nicht selten um universalistische Erwartungen zurückzuweisen. Kulturelle Differenzen sind hier keine rein kulturwissenschaftliche Beobachtungsfigur, sondern ein symbolischer Marker, der selbstreflexiv von den Akteuren eingesetzt wird, wie es zuvor bereits für die Lebensstil-Gruppen galt, so wie sie die kulturalisierte Sozialstrukturanalyse der 1980er Jahre interessierte. Neben sozialen Gruppen betrachten sich jedoch auch Institutionen immer häufiger als ,kulturell konstituiert': Dies gilt etwa für die Selbstperspektivierung von Organisationen als Ort einer gestaltbaren, organizational culture' wie auch von postmodernen Städten als Ort eines ,cultural planning'.

Die wissenschaftliche Beobachterin - Lentz und andere Ethnologinnen und Ethologen betonen dies zu Recht - kann diesem Entwenden und Umfunktionalisieren der vorgeblich eigenen Begriffe durch den Gegenstand (wobei diese bei näherer Betrachtung nie ,eigene' Begriffe waren) gegenüber nicht gleichgültig bleiben. Warum soziale Phänomene, in terms' von Kultur beschreiben, wenn die beteiligten Gruppen und Institutionen dies bereits selber tun? Führt dies nicht zu einer Verdopplung der Perspektiven und entwertet jenen Verfremdungseffekt bezüglich ihres Gegenstandes, den die Kulturwissenschaften einmal proklamiert haben? Dass einige Autoren innerhalb der Ethnologie konsequent zu diesem drastischen Schritt der Abschaffung des ,essentialisierenden' Kulturkonzeptes aufrufen, mag politisch nachvollziehbar sein, aber ich würde mit Carola Lentz übereinstimmen, dass es nicht um einen Verzicht auf eine kulturtheoretische Perspektive gehen kann, sondern vielmehr um deren nach-essentialistische Reformulierung.

Generell kann eine Popularisierung und Politisierung humanwissenschaftlich relevanter Begriffe nicht bedeuten, sie vollständig zu verbannen: Das Arsenal verfügbarer Konzepte würde sich rasch entleeren. Schließlich ist die Strategie der Kulturalisierung in der populären 
und politischen Semantik auch nicht die einzige verbreitete Tendenz. Ihr laufen mittlerweile mindestens drei weitere Verquickungen von populären und wissenschaftlichen Vokabularen zur Beschreibung und Erklärung menschlichen Verhaltens parallel, die allesamt die jeweiligen humanwissenschaftlichen Beschreibungsformen unter einen Populismusverdacht setzen, dem nur mit entsprechender theoretischer Differenzierung zu entkommen ist: eine verbreitete populäre und politische Ökonomisierung menschlichen Verhaltens, welches dieses ,wie natürlich' als Konstellation von Wahl und Entscheidung, von Konkurrenz und Markt beschreibt (vgl. auch Bröckling u.a. 2000); eine populäre Psychologisierung - auf die jüngst noch einmal Eva Illouz (2009) hingewiesen hat -, welche voraussetzungsvolle psychologische Kategorien wie Selbstentfaltung, emotionale Kompetenz etc. in die Alltagssemantik und Selbstinterpretationen der Akteure infiltriert; schließlich eine ubiquitäre Naturalisierung menschlichen Verhaltens, beeinflusst von einer Pop-Neurowissenschaft, welche Verhaltensdifferenzen über den Rückgriff auf Denkfiguren aus der Neurophysiologie, der Evolutionsbiologie und -psychologie zu erklären versucht (vgl. Brockman 2003). In allen vier diskursiven Kontexten - Kulturalisierung, Ökonomisierung, Psychologisierung, Naturalisierung - findet eine Reifizierung der jeweiligen humanwissenschaftlichen Kategorien statt.

Was die Kulturalisierungsstrategien angeht, so müssen zeitgemäße Kulturtheorien im Verhältnis zu den populären, essenzialisierenden Kulturdiskursen, die nicht selten an Elemente traditioneller Kulturphilosophien wie Herders ,Kugelmodell' anknüpfen, anders aufgebaut sein, wenn sie zum heuristisch fruchtbaren Analyseinstrument taugen wollen. Kultur ist hier weder auf eine Sphäre von Ideen, von gemeinsamen Werten und Normen zu beschränken noch im Sinne jenes kollektiven ,spirit', wie ihn Sapir in seiner klassischen Kulturanthropologie anspricht, homogenisierend auf Großgruppen zu beziehen, denen dann strikte kulturelle Grenzen nach außen zugeschrieben werden. Die Frage nach einem zeitgemäßen kulturtheoretischen Analyserahmen kann nun primär theoriehistorisch oder theoriesystematisch angegangen werden. Carola Lentz' Artikel wirft beide Fragen auf: zum einen die wissenschafts- und theoriehistorische Frage nach dem Stellenwert von Kulturtheorien und -begriffen im Kontext der Disziplinengeschichte der Ethnologie und Soziologie; zum anderen die theoriekonstruktive Frage, wie eine zeitgemäße Kulturanalyse beschaffen sein kann, welche das Kulturelle nicht auf Kosten des Sozialen profiliert.

Lentz' Artikel verweist auf die Ungleichzeitigkeit der Theorie- und Begriffskontroversen in der Wissenschaftsgeschichte miteinander zusammenhängender, aber trotzdem unterschiedlicher Disziplinen, hier: der Ethnologie und der Soziologie - eine Ungleichzeitigkeit, die gerade vor dem Hintergrund zunehmend gewünschter Interdisziplinarität ins Bewusstsein rücken sollte, um Missverständnisse zu vermeiden. Natürlich hat der Kulturbegriff in den Wissenschaftsgeschichte der Ethnologie einen ganz anderen Stellenwert, als ihm in der Soziologie zukommt. Die Ethnologie ist zumindest in einem wichtigen Segment des Faches immer schon - seit den 1870er Jahren -Kulturanthropologie gewesen und hat dabei häufig ein homogenisierendes, idealistisches Kulturverständnis transportiert. ,Kultur' kann im Rahmen des ethnologischen Denkens somit nicht per se einen Neuigkeitswert beanspruchen, sondern scheint im Gegenteil in einer bedenklichen, totalisierenden Begriffstradition zu stehen. In der Soziologie hingegen konnte sich (trotz aller Reminiszenzen einer soziologischen Kulturwissenschaft in Deutschland der Jahrhundertwende) der , interpretative turn' mit der interpretativen Mikrosoziologie nebst ihren qualitativen Methoden und der ,cultural turn' im Zuge der Semiotik, des Strukturalismus und Poststrukturalismus erst seit den 1970er Jahren als alternative, zunächst minoritäre Kraft gegen das positionieren, was Giddens sicher ein wenig pauschal den soziologischen ,orthodoxen Konsens' (von quantitativer Forschung, middle range theory und Funktionalismus) genannt hat. Diese neuen interpretativen und kulturtheoretischen Ansätze sind grundbegrifflich anders aufgebaut als der Kulturessenzialismus der klassischen Kulturanthropologie und ihnen kommt innerhalb des immanenten Differenzensystems der Disziplin So- 
ziologie damit ein anderer Stellenwert zu, als dies für das Kulturkonzept innerhalb der Ethnologie gilt (vgl. Reckwitz 2000). Statt auf die komplexe Ungleichzeitigkeit der beiden Wissenschaftsgeschichten näher einzugehen, die das Gespräch zwischen Ethnologie und Soziologie verkompliziert, möchte ich mich im Folgenden auf den theoriesystematischen Fragekomplex konzentrieren, den Carola Lentz aufwirft: die Frage nach dem Verhältnis zwischen dem Kulturellen und dem Sozialen. Die Warnung vor der ,Kulturalisierung' und der ,Entsoziologisierung', die Lentz ausspricht, wirft das Problem auf, wie sich tatsächlich ,das Soziale' und ,das Kulturelle' voneinander unterscheiden lassen und wie beide Begriffe wiederum sinnvoll aneinander gekoppelt werden können.

Die Differenz zwischen dem Kulturellen und dem Sozialen ist durchaus prekär. Dies wird schon in Carola Lentz' Artikel selbst deutlich: Sie fordert eine Resoziologisierung der Ethnologie ein, aber hat Schwierigkeiten, wirklich ,das Soziale' präzise gegen ,die Kultur' ins Stellung zu bringen. Letztlich ist es in ihrer Darstellung offenbar die Fokussierung des Phänomens der Macht, genauer von Machtkonflikten, die den Kern der Perspektive des Sozialen ausmachen soll, während die Kulturalisierung der sozialwissenschaftlichen Grundbegrifflichkeit zu einer Machtvergessenheit führe. Mich überzeugt dieser Hinweis auf Macht als Spezifikum des Sozialen nicht, denn auch Kultur in den gängigen Definitionen kann offensichtlich problemlos mit Machtbegriffen verknüpft werden, wie es im Umkreis der Arbeiten von Bourdieu, Foucault oder Laclau auch regelmäßig geschieht: Allein schon die Sprache - und ähnlich gebaute Differenzensysteme - übe einen stummen Zwang der Verhältnisse aus, indem sie Denkbares und Sagbares vorgibt, aber zugleich immer wieder zum Gegenstand von Definitionskonflikten avanciert.

Tatsächlich kann es fast so scheinen, als ob die Entwicklung soziologischer Kulturtheorien in den letzten Jahrzehnten das Soziale im Kulturellen aufgelöst hätte, so dass es immer schwerer fällt, das Soziale vom Kulturellen zu trennen. Das Soziale wird hier nämlich regelmäßig von vornherein mit Verweis auf Sinnphänomene definiert - Regeln, kollektives Wissen, Diskurse, Kommunikation und Interaktion in ihrer Reziprozität -, und diese Sinnphänomene sind es schließlich gerade, die den Kern des Kulturellen ausmachen. Auch die klassische Unterscheidung zwischen Sozialsystem und Kultursystem, wie sie von Talcott Parsons eingeführt wird, enthält bereits eine solche Kulturalisierung des Sozialsystems, indem dieses über den Weg ,normativer Erwartungen' definiert wird. Trotz dieser verbreiteten Auflösungsversuche des Sozialen im Kulturellen soll und kann die Position stark gemacht werden, dass sich die Begriffe des Sozialen und des Kulturellen zunächst sehr wohl voneinander unterscheiden lassen, um sie dann in einem zweiten Schritt sinnvoll miteinander verknüpfen zu können.

Um das Soziale vom Kulturellen überzeugend abzugrenzen, bietet sich ein Blick auf einen klassischen und einen aktuellen Autor an: auf Georg Simmel und auf Bruno Latour. Anders als Durkheim oder Weber mit ihrer Voraussetzung von moralischen Regeln und Sinnzusammenhängen formuliert Simmel in „Das Problem der Soziologie“ (1992) ein Verständnis des Sozialen, welches tatsächlich eine Differenz zum Kulturellen markiert. Er stellt bekanntlich strikt den ,Inhalt' der ,Form' von Vergesellschaftungen entgegen. Auf der Ebene des ,Inhalts' bewegen sich psychische und kulturelle Elemente, aber die Form des Sozialen ist davon zu unterscheiden: Es handelt sich hier um die beobachtbaren „Wechselwirkungen“ zwischen Menschen, um „Beziehungsformen“, um die Muster und die Struktur von Aktivitäten, die zwischen Menschen stattfinden. Diese lassen sich unabhängig von den kulturellen und psychischen Inhalten beobachten. Das Verständnis des Sozialen bei Latour, wie es jüngst in „Eine neue Soziologie für eine neue Gesellschaft“ (2007) deutlich wird, dehnt diese Relationen der Sozialität zum einen von menschlichen Akteuren auf Aktanten jeglicher Art aus, so dass sie sich von der Konstellation der Intersubjektivität lösen. Zum anderen dynamisiert Latour das Soziale deutlich gegenüber der statischeren Definition Simmels: Das Soziale ist hier in den 
„Assoziationen“ zwischen Aktanten platziert, es ist in deren sich miteinander verknüpfenden Aktivitäten zu sehen, Aktivitäten, in denen A auf B einwirkt, d.h. dort eine Verhaltensreaktion hervorruft. Das Soziale ist ein fluider Verknüpfungszusammenhang zwischen Akteuren und Aktanten, der sich nur zeitweise stabilisiert und verdichtet.

Es lässt sich damit zunächst ein Verständnis des Sozialen entwickeln, welches es abstrahiert von jedem Kulturellen - im weitesten Sinne des ,Sinnhaften' - versteht und dabei auch an die lateinische Bedeutung des ,gemeinsam, verbunden' anknüpft: Das Soziale ist in der Verknüpfung von Verhaltensakten zu suchen, Verhaltensakte, die sich in einem bestimmten zeitlichräumlichen Kontext abspielen oder aber über zeitliche und räumlich Grenzen hinweg stattfinden. Das Soziale setzt damit zwei Elemente voraus: material vorhandene Instanzen, die in irgendeinem Sinne ,agieren', , aktiv' sind, d.h. sich bewegen und ,einen Unterschied machen', damit effektive Aktivitäten entfalten; und ein Zusammenhang, eine Verknüpfung, der oder die zwischen diesen Aktivitäten existiert. Diese material vorhandenen Instanzen können in einem engen Verständnis Menschen sein, d.h. Körper der Spezies homo sapiens, in einem weiteren Verständnis kann es sich aber auch um andere Instanzen wie Tiere oder Artefakte handeln. ${ }^{1}$ Sozialität auch auf Tiere zu beziehen, ist hier kein derart exotischer Entschluss - man findet diese Ausweitung schon bei Tarde - und scheint letztlich nur konsequent, da das Verständnis des Sozialen ohne das Kulturelle nicht auf Sinnelemente rekurriert und damit zwischen Menschen und anderen material vorhandenen, sich bewegenden und Effekte erzielenden Instanzen keine Unterscheidung ziehen muss (es sei denn einen des analytischen oder normativen Interesses). Die ,soziale' Verknüpfung, d.h. die Kette der Effekte und Gegeneffekte - ob kurzfristig oder auf Dauer -, findet dabei streng genommen nicht zwischen diesen Instanzen, sondern zwischen ihren Bewegungsakten, ihren ,Aktivitäten' statt: das Soziale ist in der Verkettung von Aktivitäten zu sehen.

Entscheidend ist, dass das Soziale damit Dynamik und Strukturierung in sich vereinigt. Das Verhalten der sich bewegenden, agierenden Instanzen (Menschen etc.) hat unberechenbare, offene Effekte für das Verhalten anderer Instanzen, so dass sich hier dynamische, einmalige Ketten von Effekten und Rückeffekten ergeben können. Die Aktivitäten der beweglichen Materialitäten (Menschenkörper, Bücher, Maschinen, Töne etc.) und ihre Effekte auf andere können sich jedoch auch - zumindest zeitweise - stabilisieren und die Form regelmäßigen, berechenbaren Verhaltens annehmen, etwa im Sinne von Simmels sozialen Formen der Wechselwirkung. Das Soziale ist in diesem von Latour wie von Simmel beeinflussten Sinne damit nicht auf, soziale Ordnung' und nicht auf soziale Integration zu reduzieren und auch nicht auf zwischenmenschliche Beziehungen.

Dieses Verständnis des Sozialen, welches es vom Kulturellen zu unterscheiden vermag, mag auf den ersten Blick abstrakt scheinen, aber vertraute, soziale' Zusammenhänge der Soziologie wie Marx' Kapitalismus oder Durkheims Arbeitsteilung / soziale Differenzierung lassen sich genau als ein solches Soziales verstehen: der Kapitalismus als eine dynamische Sequenz von globalen Effekten zwischen Arbeitskräften, Gütern, Kapitalmitteln, Maschinen etc.; die Arbeitsteilung und soziale Differenzierung als Etablierung spezialisierter und schon ihrer äußeren Form voneinander abgrenzbaren Aktivitäten, die gleichwohl ineinandergreifen und miteinander zusammenhängen. Indem das Soziale damit in diesem dynamischen wie reproduktiven Zusammenhang von Verhaltensakten beweglicher Materialitäten zu verorten ist, kommt ihm per se eine ,Mächtigkeit' (die Lentz möglicherweise dazu bringt, Sozialität über Macht zu definieren) in jenem Sinne zu, dass, ein Unterschied gemacht', ein ,Effekt erzielt' wird: Im

1 Die Diskussion, inwiefern diese Instanzen als gleichberechtigt betrachtet werden sollen oder nicht, d.h. ob eine ,symmetrische Anthropologie' oder eine weiterhin asymmetrische Beschreibungsform präferiert werden soll, kann hier nicht geführt werden. In dem Moment, indem Kultur ins Spiel kommt, wird sich m.E. eine Asymmetrisierung ergeben. 
Raum der miteinander zusammenhängenden beweglichen Materialitäten werden ständig Elemente verdrängt; gleichzeitig wird aktiv etwas produziert und in die Welt gesetzt.

Versteht man das Soziale in diesem Sinne, wird es möglich, es vom Kulturellen zu unterscheiden. Folgt man zunächst einer durchaus konventionellen Begrifflichkeit, dann lässt sich das Kulturelle auf der Ebene von Sinnphänomenen ausmachen, von Regeln, die den Effekt von ,Sinn' produzieren. Letztlich bleibt die menschliche Sprache das Paradigma für ein solches Verständnis des Kulturellen: ein System von Regeln, die es erlauben, etwas als etwas zu interpretieren. Kultur ist dann ein Ort sinnhafter Repräsentationen, in denen die Dinge auf eine bestimmte Weise identifiziert, klassifiziert und bewertet und auch in eine narrative oder imaginäre Form gebracht werden.

In diesem sehr abstrakten Sinne ist das Soziale zunächst nicht mit dem Kulturellen identisch und umgekehrt. Es sind vielmehr Beschreibungsformen denkbar, die im Extrem das eine in den Blick nehmen, ohne das andere zu berühren (wobei es sich allerdings um Extremformen handelt, die real nur begrenzt vorkommen). Man kann versuchen, das Kulturelle, verstanden als menschliche Ideen- und Vorstellungswelten bzw. als konstitutive Regeln der Bedeutungsproduktion, radikal separiert von jeglichem Verhalten zu fokussieren - in Form einer Analyse ,geistiger' Phänomene, etwa in der Kunst oder Religion oder auch in populären Narrativen, Imaginationen etc. Dies wäre in der Tat ein klassisch geisteswissenschaftliches Verfahren zur Analyse von ,Sinnwelten'. Eine ,Entsoziologisierung' würde dann bedeuten, die Analyse der Dynamik und Reproduktivität von Materialitäten, insbesondere menschlichen Körpern, die in ihrem Verhalten einen Unterschied machen, zu ersetzen durch eine Analyse dieser Sinnwelten. Umgekehrt ergäbe sich eine Entkulturalisierung im Rahmen einer Beobachtungsperspektive, die quasi-behavioristisch oder artefaktsoziologisch die sich bewegenden, miteinander zusammenhängenden Materialitäten ohne jegliche Referenz auf Muster und Dynamiken dieser (mit-)strukturierenden Repräsentationssysteme in den Blick nimmt. Im Extrem stehen einander dann Kulturalismus und Soziologismus / Materialismus gegenüber.

Die Herausforderung besteht nun darin, das Soziale und das Kulturelle als zwei Seiten der gleichen Medaille wahrzunehmen oder besser: als etwas, was sich immer schon auf der gleichen Ebene des dynamisch Gegebenen bewegt. Das Soziale ist in der Tat zu kulturalisieren, aber zugleich ist das Kulturelle zu soziologisieren und damit in einem spezifischen Sinne zu materialisieren: im Sinne einer Beobachtung der in ihren Aktivitäten miteinander zusammenhängenden Materialitäten. Mir scheint die heuristische Attraktivität einer ,praxistheoretischen' Beschreibungsform - im Unterschied zu den klassischen Handlungstheorien, zu den Systemtheorien oder zum klassischen Strukturalismus - nicht zuletzt darin zu bestehen, dass sie von vornherein in ihre Begrifflichkeit diese Kopplung von Sozialem und Kulturellem versucht und darin gerade nicht einseitig, idealistisch' orientiert ist, sondern auf ihre Weise zugleich eine Version des ,cultural materialism' liefert (vgl. Reckwitz 2006, 2008). Das Konzept der - sozialen und zugleich kulturellen - Praktiken setzt zunächst an der Verstreutheit und Koordiniertheit miteinander raum-zeitlich zusammenhängender Aktivitäten an, in denen menschliche Körper miteinander und mit Artefakten dynamisch oder rigide verknüpft sind. ${ }^{2}$ Das Soziale ist hier gerade nicht in jenem verkürzten, sogleich schon kulturalisierten Sinne von Normen, Werten, Intersubjektivität oder Kommunikation zu verstehen, sondern in den Effekten und einseitigen oder wechselseitigen Rückeffekten der Aktivitäten von menschlichen Körpern sowie von Artefakten: es ist materialisiert. Zugleich jedoch ist dieses Soziale ,immer schon' als kulturalisiert zu verstehen: Kultur kann praxeologisch als Ensemble von Wissensformen reformuliert werden, und diese Wissensformen sind immer schon im reproduktiven wie im dy-

2 Es wäre sinnvoll, in diesem Sinne, Aktivitäten' und ,Praktiken' voneinander zu unterscheiden: Aktivitäten können sich in die Routineform von sozial-kulturellen Praktiken verfestigen, müssen dies aber nicht zwangsläufig. 
namisch-unberechenbaren Verhalten menschlicher Körper enthalten, in deren Rahmen solche Körper auch zu Akteuren bzw. Subjekten avancieren. Der Begriff, Wissen' wird hier von vornherein auf praktisches Verhalten bezogen, in einem ermöglichenden wie einschränkenden Sinne. Umgekehrt ist damit Kultur im Sinne dieser Wissensordnungen als von vornherein soziologisiert / materialisiert zu denken: als ein Wissen, das entweder in den menschlichen Körpern inkorporiert ist und von dort aus das Verhalten als ,tacit knowledge' strukturiert oder aber das auf die Trägerschaft durch Artefakte (Filme, Bücher, Kleidungsstücke etc.) angewiesen ist - bis hin zur Trägerschaft durch von der menschlichen Stimme produzierten Töne im Falle der mündlichen Kommunikation. Auch die Zeichen und Zeichensequenzen, welche die Semiotik interessieren, kommen damit von vornherein als eine material-kulturelle Doppelstruktur in den Blick: Als Zeichenformen sind sie genau so material wie Körper und andere Artefakte; als Schriftzeichen, Töne, Bilder, Filme etc. sind sie Teil der zirkulierenden, ,sozial' miteinander verknüpften Entitäten. Als Zeicheninhalte / Bedeutungen, d.h. auf der Ebene des Sinns, der diesen Schriftzeichen, Tönen, Bildern vor dem Hintergrund eines impliziten Wissens von Rezipienten zugeschrieben werden, sind sie zugleich kulturell.

Als entscheidend für eine solche kulturtheoretische und ebenso materialistische Perspektive, die in diesem Sinne von sozial-kulturellen Praxis ausgeht, stellt sich heraus, dass sie sich mit einem zweiten Dualismus produktiv auseinandersetzt, der bei Lentz angesprochen wird: jenem zwischen Konsens und Konflikt oder besser und allgemeiner: zwischen Stabilisierung und Destabilisierung von Strukturen. Das Problem der alten Kulturanthropologie von Sapir, Benedict und anderen besteht nicht nur darin, dass ihre, idealistisch' orientierte, immanente Beschreibung von Repräsentationssystemen die genaue Analyse der Struktur und Dynamik sozialer Verhaltenskomplexe, damit des Sozialen, vernachlässigt. Das zweite Problem ist in einer Homogenitätsperspektive zu suchen, die davon ausgeht, dass diese Repräsentationssysteme zudem immanent geordnet und widerspruchsfrei strukturiert sind und damit auch eine fixe Grenze nach außen, zu anderen, Kulturen' bilden. Eine solche strukturalistische Präjudizierung von Geordnetheit und unendlicher Reproduktion ist jedoch kein potentielles Problem nur der Kulturtheorien. Sowohl kulturalistische als auch soziologistisch-materialistische Ansätze können einen strukturalistischen Bias enthalten. So kann auch eine Fixierung auf das Soziale ganz jenseits der Kultur mit einer Voraussetzung stabiler Wechselwirkungen und sozialer Gesetzmäßigkeiten verbunden sein, so wie umgekehrt kulturalistische Perspektiven, die auf Zeichensequenzen fixiert sind, post-strukturalistisch gerade die immanente Dynamik und Unberechenbarkeit dieser Sequenzen betonen können.

Gefragt ist damit eine kulturtheoretische und materialistische Theorieperspektive, die sowohl die relative Ungeordnetheit und Veränderungsoffenheit von Praktiken und ihrer Wissensformen als auch deren temporäre Stabilisierungen und Reproduktionen begreift und damit jenen Essentialismus hinter sich lässt, den auch der populäre Kulturdiskurs teilweise enthält. Aus meiner Sicht kann man von manchen post-strukturalistischen Ansätzen lernen, diesem Kulturessentialismus - ebenso wie einem analogen soziologistischen Strukturessentialismus - systematisch zu entgehen, ohne sich mit einem bloßen ,Mittelweg' zu begnügen. ${ }^{3}$ Stattdessen können sozial-kulturelle Stabilisierungen als temporäre Verdichtungen innerhalb von Prozessen der Destabilisierung verstanden werden. Die potentielle und faktische Dynamik und Unberechenbarkeit der Aktivitäten und Assoziationen, die menschliche Körper, Artefakte, Zeichen und Wissen besitzen, wird immer wieder durch reproduktive Praktiken- / Wissenskomplexe stabilisiert. Aber diese Stabilisierungen - sei es in Institutionen und Technologien, sei es in sozialen Gruppen, Lebensformen oder Subjektivierungen - sind selber temporäre Gebilde, die wiederum durch Verhaltensakte und Effekte, welche nicht in die Praktiken / Wissens-

3 Am offensivsten wird eine solche post-strukturalistische Theorie des Sozialen in der gegenwärtigen Diskussion bei Latour (2007) skizziert. 
ordnungen , hineinpassen', und durch unberechenbare Sinneffekte einander überlagernder oder widersprechender Wissensformen gesprengt werden können.

Carola Lentz' vom symbolischen Interaktionismus beeinflusster Vorschlag, Kultur auf der Ebene von „Aushandlungspraktiken“ mit jeweils offenem Ausgang festzumachen, scheint dadurch motiviert, genau diese Ergebnisoffenheit von und Konkurrenz um Repräsentationssysteme zu fokussieren. Letztlich scheint mir das Konzept der Aushandlungspraktiken jedoch rationalistisch verengend: Kultur wird hier primär als ein Objekt reflektierter Auseinandersetzung verstanden, und dieser bewusste Umgang mit der Kultur als Objekt wird mit einer politisch-ökonomischen und zudem intersubjektiv orientierten Metapher, der ,Aushandlung' / ,Verhandlung', belegt. Ein praxeologisches Kulturverständnis versteht Kultur dagegen primär als ein implizites, in mancher Hinsicht präreflexives Wissen, das in jeglichen sozial-kulturellen Praktiken inkorporiert ist. Jene Praktiken, in denen Wissen oder Zeichen speziell und explizit zum , Thema' werden, stellen sich darin als eine Untermenge dar (was heißt, das eben auch in diesen Praktiken nicht alles zum Thema wird). Diese Thematisierung von Zeichen und Wissen kann nun jedoch wiederum sehr unterschiedliche Formen annehmen. Zum einen kann sie verschiedenste mediale Träger-Bild, Schrift, Stimme - umfassen. Die Thematisierung von Kultur muss hier keineswegs unbedingt eine diskursive Form erhalten (, diskursiv' im Sinne von Habermas, nicht von Foucault), wie sie die Rede von der , negotiated order' suggeriert, sondern kann etwa auch eine narrative oder imaginär-bildhafte Struktur besitzen. Zudem kann es sich natürlich in jenen Fällen, in denen tatsächlich unterschiedliche Akteure oder Akteursgruppen mit unterschiedlichen Wissens- und Repräsentationsformen einander gegenüberstehen, tatsächlich um Konstellationen handeln, die Praktiken der friedlich-reziproken Aushandlung folgen. Es können aber ebenso Konstellationen des Kampfes um die einseitige Durchsetzung kultureller Modelle vorliegen. Die symbolisch-interaktionistische (zudem artefaktvergessene) Sicht verengt den Blick auf das Kulturelle damit bedenklich auf ein sehr enges Segment, nämlich auf den der offenen, expliziten, verhandlungsförmigen Auseinandersetzung mit Kultur, und ist dabei selber erstaunlich machtvergessen.

Welcher Ort kann der Dimension der ,Macht' nun innerhalb einer solchen praxeologischen Perspektive auf Kulturelles und Soziales zukommen? Carola Lentz fordert dieses Konzept zu Recht ein. Heuristisch am interessantesten scheint mir, jenseits einer Verengung von Macht auf spezifische Praktiken und Felder - wie man es etwa in der Systemtheorie findet -, aber auch der differenzierungslosen Annahme einer Ubiquität von Macht, wie sie manche FoucaultAnhänger präferieren, unterschiedliche Formen von Macht in ihrer Wirkung innerhalb des Sozialen / Kulturellen zu unterscheiden. Die bekannte Kontroverse zwischen akteurs- und strukturtheoretischen Machtmodellen (vgl. Lukes 1973) kann dabei produktiv verarbeitet werden. Generell lassen sich Prozesse der Macht als solche des Sich-Durchsetzens von spezifischen Verhaltensakten sowie von kulturellen Wissensformen (in Körpern oder Artefakten) begreifen. Dies gilt damit unabhängig von jenem sich aufzwingenden subjektiven Willen von Akteur A auf B, wie ihn Max Weber in seinem klassischen, handlungstheoretischen Machtverständnis voraussetzte, und ist ebenso wenig reduziert auf das Wirken fixer Strukturen oder Regelsysteme. Das Sich-Durchsetzen enthält ein Moment der Vervielfältigung wie auch der Reduktion: Es entstehen und multiplizieren sich neue und andersartige Aktivitäten (menschliche, artefaktförmige, ,natürliche' etc.) sowie neue und andere Wissensformen (in menschlichen Körpern, in der Zeichenzirkulation, in kulturellen Objekten), zugleich können dabei andere Aktivitäten und Wissensformen verdrängt, damit Existenzmöglichkeiten reduziert werden.

Die Konstellationen des Sich-Durchsetzens unterscheiden sich jedoch im Falle jener verhältnismäßig ,flüssigen', entstrukturierenden und destabilisierenden Aggregatformen des Sozialen / Kulturellen, in denen sich neue Aktivitäten und Wissenselemente aneinander knüpfen 
und zirkulieren, und jenen relativ ,festen', strukturierenden und stabilisierenden Aggregatformen des Sozialen / Kulturellen voneinander, in denen sich Blöcke und Matrizen von Praktiken und Wissensordnungen reproduzieren. In letzterer Konstellation manifestiert sich Macht in der Reproduktion eines Herrschaftssystems, das gleichförmig (allerdings nie völlig identisch) Verhalten hervorbringt, in Form von Kontrolle und Zwang, zugleich aber auch einer massenhaften Ermöglichung konzentrierter, berechenbarer Prozesse. Kulturell kann eine solche Konstellation über Hegemonien und Inkorporierungen / Subjektivierungen stabilisiert werden. In der erstgenannten Konstellation manifestiert sich hingegen Macht im ,Können’ (pouvoir) und Vermögen neuer Möglichkeiten des Verhaltens und Wissens und zugleich in der agonalen Struktur des Kampfes zwischen verschiedenen neuen oder alten Verhaltens- und Wissensformen um ihre Faktizität und Legitimität. Eine allgemeine Theorie der Macht wäre dann zu ersetzen durch eine Analytik der - auch historisch - unterschiedlichen Formen des SichDurchsetzens in verschiedenen Praxis-Konstellationen und ganzen Gesellschafts- / Naturformationen. Ob dies dann noch ,Kulturtheorie' zu nennen wäre, ist möglicherweise eine eher sekundäre Frage.

\section{Literatur}

Bal, Mieke (2002): Travelling Concepts in the Humanities, Toronto.

Bröckling, Ulrich / Susanne Krasmann / Thomas Lemke (Hrsg.) (2000): Gouvernementalität der Gegenwart. Studien zur Ökonomisierung des Sozialen, Frankfurt / Main.

Brockman, John (Hrsg.) (2003): The New Humanists. Science at the edge, New York.

Illouz, Eva (2009): Die Errettung der modernen Seele, Frankfurt / Main.

Latour, Bruno (2007): Eine neue Soziologie für eine neue Gesellschaft. Einführung in die Akteur-Netzwerk-Theorie, Frankfurt / Main.

Lukes, Steven (1974): Power: A Radical View, London.

Reckwitz, Andreas (2000): Die Transformation der Kulturtheorien. Zur Entwicklung eines Theorieprogramms, Weilerswist.

Reckwitz, Andreas (2006): Das hybride Subjekt. Eine Theorie der Subjektkulturen von der bürgerlichen Moderne zur Postmoderne, Weilerswist.

Reckwitz, Andreas (2008): Grundelemente einer Theorie sozialer Praktiken. Eine sozialtheoretische Perspektive, in: Ders., Unscharfe Grenzen. Perspektiven der Kultursoziologie, Bielefeld, S. 97-130.

Simmel, Georg (1992): Das Problem der Soziologie, in: Ders., Soziologie. Untersuchungen über die Formen der Vergesellschaftung, Frankfurt / Main, S. 13-62.

Prof. Dr. Andreas Reckwitz Universität Konstanz Lehrstuhl für Allgemeine Soziologie und Kultursoziologie Universitätsstraße 10 78457 Konstanz Andreas.Reckwitz@uni-konstanz.de 